\title{
Norvaline Reduces Blood Pressure and Induces Diuresis in Rats with Inherited Stress-Induced Arterial Hypertension
}

\author{
Michael A. Gilinsky, ${ }^{1}$ Yulia K. Polityko, ${ }^{2}$ Arkady L. Markel, ${ }^{2,3}$ Tatyana V. Latysheva, ${ }^{1}$ \\ Abraham O. Samson (iD, ${ }^{4}$ Baruh Polis ${ }^{(D)}{ }^{4}$ and Sergey E. Naumenko' \\ ${ }^{1}$ Scientific Research Institute of Physiology and Basic Medicine, 4 Timakova Street, Novosibirsk 630117, Russia \\ ${ }^{2}$ Federal Scientific Center Institute of Cytology and Genetics, 6, Prosp. M. A. Lavrent'eva, Novosibirsk, Russia \\ ${ }^{3}$ Novosibirsk State University, Novosibirsk, Russia \\ ${ }^{4}$ Drug Discovery Laboratory, The Azrieli Faculty of Medicine, Bar-Ilan University, Safed, Israel
}

Correspondence should be addressed to Baruh Polis; baruhpolis@gmail.com

Received 21 June 2019; Accepted 24 December 2019; Published 14 February 2020

Academic Editor: Fabrizio Montecucco

Copyright (c) 2020 Michael A. Gilinsky et al. This is an open access article distributed under the Creative Commons Attribution License, which permits unrestricted use, distribution, and reproduction in any medium, provided the original work is properly cited.

\begin{abstract}
Growing evidence suggests that increased arginase activity affects vital bioprocesses in various systems and universally mediates the pathogenesis of numerous metabolic diseases. The adverse effects of arginase are associated with a severe decline in L-arginine bioavailability, which leads to nitric oxide synthase substrate insufficiency, uncoupling, and, eventually, superoxide anion generation and substantial reduction of nitric oxide (NO) synthesis. In cooperation, it contributes to chronic oxidative stress and endothelial dysfunction, which might lead to hypertension and atherosclerosis. Recent preclinical investigations point arginase as a promising therapeutic target in ameliorating metabolic and vascular dysfunctions. In the present study, adult rats with inherited stress-induced arterial hypertension (ISIAH) were used as a model of hypertension. Wistar rats served as normotensive controls. Experimental animals were intraperitoneally administered for seven days with nonproteinogenic amino acid L-norvaline (30 mg/ $\mathrm{kg}$ /day), which is a potent arginase inhibitor, or with the vehicle. Blood pressure (BP), body weight, and diuresis were monitored. The changes in blood and urine levels of creatinine, urea, and NO metabolites were analyzed. We observed a significant decline in $\mathrm{BP}$ and induced diuresis in ISIAH rats following the treatment. The same procedure did not affect the BP of control animals. Remarkably, the treatment had no influence upon glomerular filtration rate in two experimental groups, just like the daily excretion of creatinine and urea. Conversely, NO metabolite levels were amplified in normotonic but not in hypertensive rats following the treatment. The data indicate that L-norvaline is a potential antihypertensive agent and deserves to be clinically investigated. Moreover, we suggest that changes in blood and urine are causally related to the effect of L-norvaline upon BP regulation.
\end{abstract}

\section{Introduction}

Hypertension is a serious, continuously growing healthcare problem. The number of people suffering from high blood pressure (BP) has doubled over the last 40 years afflicting more than 1.13 billion people worldwide [1], including 75 million individuals in the United States alone [2]. The disease represents a leading mortality cause, with more than 7.6 million deaths per annum [3]. Moreover, there is a robust causality between devastating cardiovascular diseases, including myocardial infarction and stroke, and raised BP [3].

The hypertension etiology remains ambiguous. Recently disclosed various behavioral and genetic factors do not explicitly clarify the precise mechanisms of hypertension development. However, growing evidence indicates psychosocial factors as having an essential causative role [4]. Particular, the role of emotional stress in hypertension etiology is well established [5]. Additionally, recent empirical data point to endothelial dysfunction and reduced 


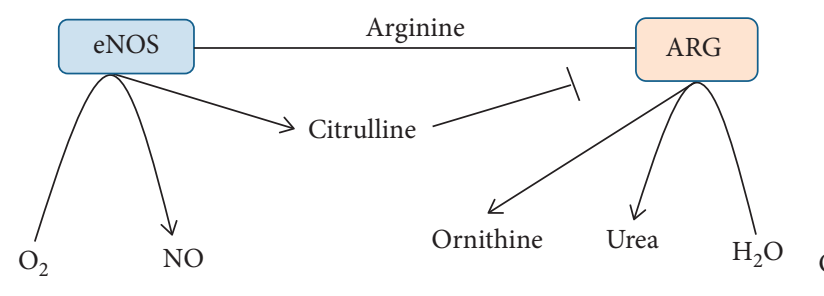

(a)

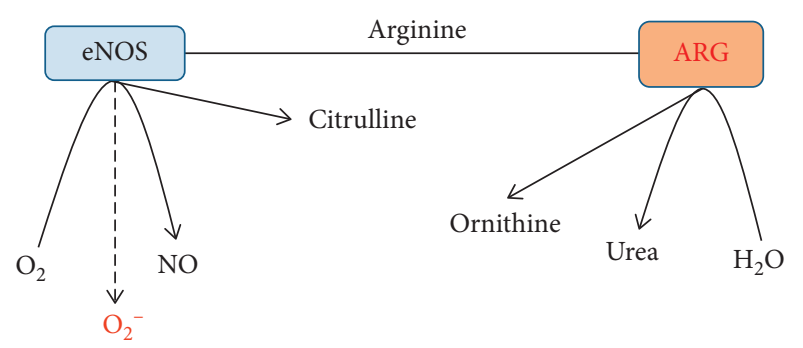

(b)

FIgURe 1: Metabolic fates of arginine in the mammalian cells. (a) Arginine is a mutual substrate for arginase and NOS, which are in equilibrium in physiologic conditions. Regularly coupled eNOS utilizes $\mathrm{O}_{2}$ and arginine to produce NO and citrulline. Arginase, in turn, converts arginine into ornithine and urea. (b) eNOS is uncoupled by substrate deficiency to produce superoxide anion rather than NO, which further diminishes NO availability.

nitric oxide (NO) bioactivity as the leading pathophysiological abnormalities associated with hypertension [6]. Remarkably, L-arginine supplementation reduces systemic blood pressure (BP) in some forms of experimental hypertension $[7,8]$ due to its direct effect upon NO synthesis and characteristic antioxidant activities, which regulate blood pressure via redox-sensitive proteins [9]. It was suggested that supplemental L-arginine is more effective in salt-sensitive hypertension than in essential hypertension [10].

Of note, L-arginine is a semiessential amino acid, which is obtained from natural dietary sources and can be produced endogenously in various organs [11]. L-arginine plays a vital role in various physiological functions and, prominently, in maintaining vascular homeostasis [10]. L-arginine is a mutual substrate for arginase and nitric oxide synthase (NOS) (Figure 1(a)). Arginase is a manganese-containing enzyme that converts L-arginine into L-ornithine and urea. NOS isoforms, in turn, catalyze the production of NO and citrulline. Recent data suggest that NO-mediated vasodilation is substantially inhibited in hypertension due to an increase in arginase activity in endothelial cells (EC), which limits L-arginine availability to NOS for NO production [12].

The main effects of arginase upon BP are associated with inhibition of the NO synthesis, which is a potent vasodilator [13]. Of note, NO represents a central EC protective factor, under physiological conditions [14]; however, it becomes deleterious under oxidative stress. A decline in L-arginine bioavailability leads to endothelial NOS (eNOS) uncoupling and deflection from regular NO synthesis toward superoxide ion generation (Figure 1(b)) [15]. Remarkably, eNOS-deficient mice demonstrate an increased rate of atherosclerosis [16], in part, due to elevated BP [17].

Two arginase isoforms have been identified. Both types, cytoplasmic arginase I (ARG1) and mitochondrial arginase II (ARG2), have been shown to inhibit the NO production by regulating the L-arginine bioavailability [18]. While L-arginine affinity for NOS is more than 1,000 times higher than for arginase, arginase is about 1,000 times more active than NOS, which provides the equilibrium in L-arginine utilization in physiologic conditions [19]. However, the decline in substrate bioavailability and/or activation of arginase leads to a substantial shift of the balance toward ornithine synthesis (Figure 1(b)).

It seems that arginase activation is a conserved evolutionary reaction to various stimuli [20]. Arginase expression is inducible by catecholamines, cytokines, lipopolysaccharide, tumor necrosis factor, oxidized low-density lipoprotein (OxLDL), and hypoxia [21]. Remarkably, human and rodent aortic EC exposure to OxLDL is followed by a rapid increase in arginase activity, which is associated with ARG2 translocation from the mitochondria to the cytosol of EC [22]. Of note, hypertensive men show significantly elevated OxLDL levels compared to normotensives [23], which suggests a connection between hypertension, atherosclerosis, and arginase activation.

EC dysfunction, arising from the decline in L-arginine bioavailability and violation of the normal NOS function (in which arginase plays a major role), leads to the development of numerous cardiovascular pathologies, and, particularly, the development of arterial hypertension. Therefore, arginase inhibition has been proposed as a therapeutic approach for the treatment of cardiovascular diseases, including hypertension [24]. Incidentally, manipulating with arginase expression levels and its activity became the goal of many studies aimed to disclose the precise mechanisms of hypertension and find ways to combat this deleterious disease.

El-Bassossy et al. demonstrated that arginase inhibition alleviates fructose-induced hypertension in a rat model of metabolic syndrome [25]. The authors gavaged the experimental animals with solutions of L-citrulline, L-norvaline $(50 \mathrm{mg} / \mathrm{kg} /$ day $)$, and L-ornithine for six weeks. Of note, the inhibitors utilized possess different modes of arginase inhibitory activity. The authors suggest that the effects of the arginase inhibition are directly mediated via NO signaling protection and endothelial-dependent relaxation, while indirectly associated with insulin sensitivity improvement. Another recent study by Peyton et al., utilizing Zucker rats as a model of obesity, evidenced a significant effect of a sustained intraperitoneal infusion of the arginase inhibitor $\mathrm{N} \omega$-hydroxy-nor-l-arginine $(25 \mathrm{mg} / \mathrm{kg} /$ day) for four weeks on systolic BP [26]. Of note, Zucker rats display elevated $\mathrm{BP}(\approx 140 \mathrm{mmHg})$ from the age of 12 weeks, and application of arginase inhibitor or L-arginine has a 
similar BP-reducing effect. The same arginase inhibitor $(40 \mathrm{mg} /$ day) for ten weeks was used by Bagnost et al. in spontaneously hypertensive rats [27]. The authors speculate that the antihypertensive effect of arginase inhibition is associated with modulation of mesenteric artery reactivity, restoration of angiotensin-II-induced contraction, and acetylcholine-induced vasodilation. Another study by Pokrovskiy et al. convincingly demonstrated that application of an arginase inhibitor L-norvaline $(10 \mathrm{mg} / \mathrm{kg} / \mathrm{day})$ precludes the endothelial dysfunction development in a rat model of methionine and N-nitro-L-arginine methyl esterinduced NO deficiency [28].

Of note, L-norvaline is a potent arginase inhibitor [29] and a unique compound with a broad spectrum of biological properties. It acts via negative feedback inhibition mechanism due to its structural similarity to ornithine [30] and substantially amplifies the NO production rate [31]. Moreover, in contrast to other arginase inhibitors, L-norvaline inhibits ornithine transcarbamylase (OTC), which converts ornithine to citrulline in the mitochondria [32]. The association of OTC gene polymorphisms with increased risk of hypertension has been well described [33]. Therefore, L-norvaline application in the treatment of hypertension might be particularly beneficial.

Additionally, L-norvaline possesses anti-inflammatory properties due to its potency of inhibiting ribosomal protein S6 kinase beta-1 (S6K1) [34, 35]. S6K1is a direct downstream target of the mechanistic target of rapamycin (mTOR). Of note, growing evidence indicates that inflammation is a key factor of the hypertension pathogenesis, since endothelial dysfunction, together with oxidative stress, is evidently involved in the inflammatory cascade $[36,37]$. Remarkably, inhibition of mTOR signaling pathway with rapamycin $(1.5 \mathrm{mg} / \mathrm{kg} /$ day $)$ has been shown to attenuate salt-induced hypertension in rats [38]. Accordingly, L-norvaline possesses several potentially antihypertensive modes of activity. It is worth mentioning that L-norvaline has already demonstrated a serious therapeutic potential in preclinical studies of various diseases with a clear metabolic signature. In particular, it has been suggested as a possible candidate to treat complications of diabetes mellitus [39] and Alzheimer's disease [40].

The purpose of the present study was to investigate the therapeutic effects of L-norvaline in a rodent model of inherited stress-induced arterial hypertension (ISIAH). The ISIAH rats represent a unique model of stress-sensitive arterial hypertension [41]. The strain has been created in the Institute of Cytology and Genetics of the Siberian Branch of the Russian Academy of Sciences using Wistar rats' background and is characterized by the genetically determined enhanced response of the neuroendocrine and renal regulatory systems to stress [42]. Consequently, the ISIAH strain is an optimal rodent model for investigation of the genetic and physiological mechanisms and pathogenesis of stress-sensitive hypertension. Particularly, we aimed to correlate the treatment-related changes in systolic BP with the blood and urine concentrations of NO metabolites in the stress-sensitive arterial hypertension.

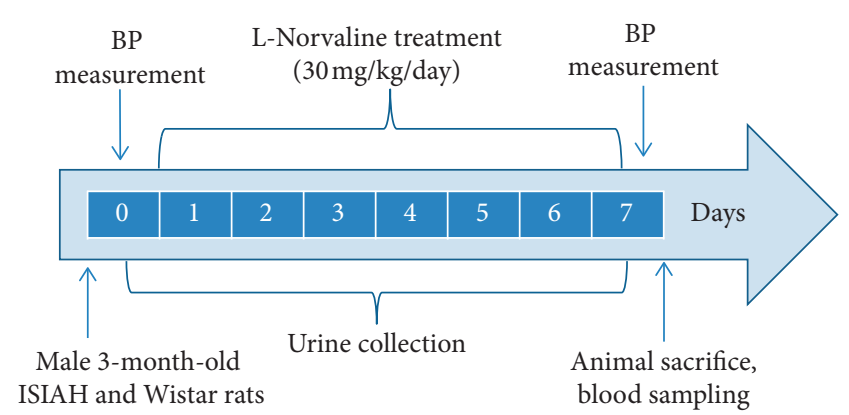

FIgURE 2: The experimental design.

\section{Materials and Methods}

2.1. Animals. Studies were approved by the Biomedical Ethics Committee of the Scientific Research Institute of Physiology and Basic Medicine (SRIPBM). The study was conducted using permit no. 7 of September 10, 2015, and following the European Community Council Directive 86/ 609/EEC. The ISIAH rat strain was developed and bred at the animal facility of the Institute of Cytology and Genetics (Novosibirsk, Russia). Wistar rats were bred in the Scientific Research Institute of Physiology and Basic Medicine (Novosibirsk, Russia) and served as normotensive controls. Male rats at the age of 12 weeks were used in all experiments.

\subsection{Experimental Design and Treatments.} Three-month-old male rats weighing about $400 \mathrm{~g}$ were randomly divided into four groups. Two control groups consisted of intact hypertensive ISIAH rats $(n=9)$ and intact normotensive Wistar rats $(n=6)$. Two experimental groups, with animals receiving L-norvaline treatment, consisted of ISIAH hypertensive $(n=8)$ and Wistar normotensive $(n=6)$ rats.

L-norvaline (Sigma, St. Louis, MO, USA) was dissolved in isotonic saline solution. The animals were administered intraperitoneally with one $\mathrm{ml}$ of L-norvaline solution daily $(30 \mathrm{mg} / \mathrm{kg} / \mathrm{day})$, or with the vehicle, at $2 \mathrm{PM}$ every day for 7 days. The animals were housed individually in the metabolic cages (Italplast, Italy), which provide separation of urine and feces through the unique design of the funnel and the separation cone. Animals had free access to balanced food and water.

Urine was collected daily at $10 \mathrm{AM}$. Blood was collected during slaughter on the eighth experimental day after decapitation on the guillotine (Figure 2). Urea in blood plasma and urine was measured using the Urea-Novo kit (VectorBest, Novosibirsk, Russia) in accordance with the company instructions, following the method we modified. The modification consisted of using a multimodal reader TriStar LB 941 (Berthold Technologies, Germany) to detect optical density instead of using a cuvette spectrophotometer. The remaining parameters of blood and urine: creatinine, uric acid, and urea nitrogen (UN) were determined by the colorimetric method using an integrated, biochemical system Dimension RxL Max (Siemens, Germany) according to the instructions of the company. 
2.3. BP Measurement. For BP measurements, rats were habituated to the RR-300 restraint system (IBI Scientific, Dubuque, Iowa, USA) for $15 \mathrm{~min} /$ day for seven days prior to the experiment.

Blood pressure (BP) was measured indirectly at 11-12 $\mathrm{AM}$ on the first and eighth days of the experiment by the same investigator. The measurements have been performed in conscious and restrained rats by the indirect tail-cuff method using a semiautomatic noninvasive BP monitoring system BIOPAC-MP system (Goleta, CA, USA) After five min of habituation in the restraint chamber, a typical series included six consequent repetitions of the automated inflation-deflation cycles. The arithmetic average value of the readings was calculated and taken as the $\mathrm{BP}$.

2.4. Glomerular Filtration Rate. Glomerular filtration rate (GFR) is a standard test utilized to evaluate the kidney function [43]. It assesses the volume of fluid filtered from the glomerular apparatus into Bowman's capsule. In general practice, GFR is estimated by use of serum and urine creatinine concentrations, which is an endogenous filtration marker [44] and typically expressed in units of volume per time. In order to calculate GFR, the ratio between the urine and plasma creatinine concentrations was multiplied by the value of urine output in one minute per 100 gram of the animals' body weight.

2.5. Nitrite/Nitrate Measurement. NO has an extremely short half-life (less than $5 \mathrm{sec}$ ) in biological fluids, which substantially limits its investigations [45]. NO is scavenged by oxyhemoglobin in blood, forming nitrate, and oxidized by a copper enzyme ceruloplasmin to produce nitrite [46]. Nitrate and nitrite are relatively stable metabolites of $\mathrm{NO}$, which stay stable for several hours in plasma [47]. In order to study the effect of L-norvaline treatment upon the levels of NO metabolites (nitrites and nitrates), blood and urine of the experimental animals have been subjected to the analysis. A commercially available reagent kit K1342 (Abnova, Taipei, Taiwan) has been used for the measurements. This colorimetric kit provides an accurate and convenient way to measure the total nitrate/nitrite concentration in a simple two-step process. The first step is the conversion of nitrate to nitrite using nitrate reductase. At the second stage, Griess reagent is added, which converts nitrite to a dark violet azo compound. Photometric measurement of optical density, due to this azo chromophore, accurately determines the concentration of $\mathrm{NO} 2$.

2.6. Statistical Analysis. Statistical analysis was conducted using SPSS version 22 (IBM, Armonk, NY, USA) for Windows. The significance was set at $95 \%$ of confidence. All the results are presented as mean with standard error. The Shapiro-Wilk test showed that the data fit a normal distribution, and Levene's test was performed to confirm equal variance between the groups being compared. The means were compared between two groups using Student's $t$-test (if appropriate) or one-way analysis of variance (ANOVA), with Tukey's multiple comparison test used for post hoc analyses. Two-way ANOVA has been applied to check if there was an interaction between two independent variables on the dependent variable. Throughout the text and in bar plots, the variability is indicated by the standard error of the mean (SEM).

\section{Results}

3.1. L-Norvaline Effectively Reduced BP in ISIAH Rats. The basal levels of systolic blood pressure measured by the indirect tail-cuff method in conscious restrained rats were $123.4 \pm 1.23(n=12) \mathrm{mmHg}$ in the Wistar and $176.5 \pm 1.61$ $(n=15) \mathrm{mmHg}$ in the ISIAH rats (Figure 3(a)), which accords with our previously published data [48]. L-norvaline administration led to a substantial drop (by about 19\%) in systolic BP in ISIAH rats (Figure 3(b)). The main effect of the treatment was very significant $F_{3,28}=54.63(p<0.0001)$ on the $7^{\text {th }}$ experimental day. The average registered BP in the ISIAH treated with L-norvaline group was $143.1 \pm 2.33 \mathrm{mmHg}$. The same treatment protocol had a minor nonsignificant effect upon the BP in Wistar rats. The reduction was about $13 \%$ or $16 \mathrm{mmHg}$; however, the effect was not statistically significant in this experimental group, which was evident by Tukey's multiple comparisons post hoc test. Two-way ANOVA revealed a significant interaction between the strain and the treatment variables on BP values $F_{(1,25)}=4.429, p=0.046$, which account for $2.271 \%$ of the total variance (Figure 3(b)).

3.2. L-Norvaline Causes Inconsequential Weight Loss in Experimental Animals. The control intact animals from two experimental strains gained about 11-12 grams on average. L-norvaline administrations for seven days led to a minor (about 5\%) loss of weight, which was about $24 \mathrm{~g}$ in ISIAH rats and $22 \mathrm{~g}$ in Wistar rats on average. However, the main effect of the treatment was not statistically significant with $p=0.38$ and $F_{3,28}=1.076$. Moreover, ANOVA with repeated measures did not reveal any significant effect of time upon the animals' body weight.

3.3. L-Norvaline Treatment Led to a Significant Decline in Urine Creatinine and Urea Concentrations in ISIAH Rats but Was Followed by opposite Metabolic Effects in Wistar Rats. There Was No Treatment-Associated Effect on the Glomerular Filtration Rate in Two Groups. The treatment with L-norvaline led to a significant drop in the urine concentration of creatinine and urea in the hypertensive ISIAH rats compared to the intact ISIAH rats. By contrast, in the Wistar rats, the level of these urine metabolites had a clear tendency to increase (Table 1). In this regard, the daily amounts of the excreted creatinine and urea, despite interstrain differences of their concentrations, on the seventh day did not differ between the ISIAH and Wistar rats, both in control and L-norvaline treated groups. Of note, the glomerular filtration rate (GFR) in the groups of intact rats and those treated with L-norvaline was the same. 


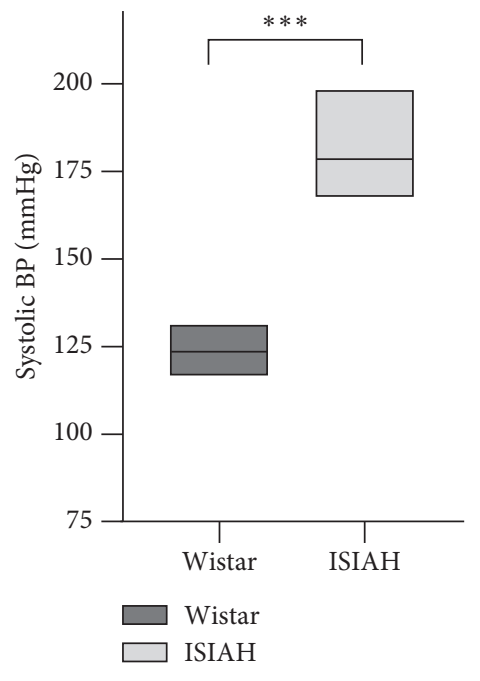

(a)

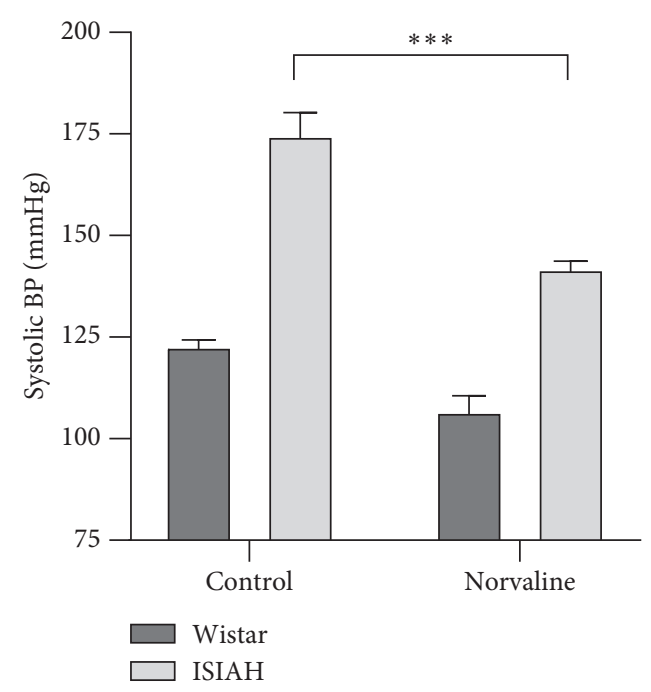

(b)

FIGURE 3: Effects of L-norvaline treatment on the systolic blood pressure (BP). (a) The mean basal levels of systolic BP measured in two experimental rat strains before treatment on day 0 . Unpaired $t$-test. Floating bars ( $\min$ to max with line at mean). (b) The mean systolic BP on day 7. Two-way ANOVA test, ${ }^{* * *} p<0.001$. Data presented as mean \pm SEM.

TABLE 1: The effects of L-norvaline on some blood and urine components in hypertensive (ISIAH) and normotensive (Wistar) rats on the seventh treatment day. Values are expressed as the mean $\pm \mathrm{SEM},{ }^{*} p<0.05$.

\begin{tabular}{|c|c|c|c|c|}
\hline \multirow{2}{*}{$\begin{array}{l}\text { Rat strain } \\
\text { Type of treatment }\end{array}$} & \multicolumn{2}{|c|}{ ISIAH } & \multicolumn{2}{|c|}{ Wistar } \\
\hline & Control & Norvaline & Control & Norvaline \\
\hline Creatinine in urine $\mathrm{mmol} / \mathrm{l}$ & $7,35 \pm 0,51$ & $5,39 \pm 0,56^{*}$ & $5,87 \pm 0,98$ & $10,08 \pm 1,13^{*}$ \\
\hline Creatinine excretion $\mu \mathrm{mol} /$ day & $95 \pm 6,0$ & $102 \pm 9,5$ & $92 \pm 2,6$ & $92 \pm 5,1$ \\
\hline Creatinine in plasma $\mu \mathrm{mol} / \mathrm{l}$ & $42,4 \pm 2,5$ & $38,8 \pm 1,4$ & $37,2 \pm 2,3$ & $37,1 \pm 1,7$ \\
\hline Glomerular filtration rate $\mathrm{ml} / \mathrm{min}$ per $100 \mathrm{~g}$ body mass & $0,42 \pm 0,05$ & $0,42 \pm 0,047$ & $0,48 \pm 0,036$ & $0,48 \pm 0,02$ \\
\hline Uric acid in urine $\mu \mathrm{mol} / \mathrm{l}$ & $496 \pm 67$ & $671 \pm 49$ & $450 \pm 91$ & $449 \pm 41$ \\
\hline Uric acid in plasma $\mu \mathrm{mol} / \mathrm{l}$ & $34,6 \pm 7,4$ & $33,8 \pm 1.3$ & $36,6 \pm 2,7$ & $41,6 \pm 8,3$ \\
\hline $\mathrm{UN}$ in urine $\mathrm{mmol} / \mathrm{l}$ & $971 \pm 4,3$ & $706 \pm 98,3$ & $919 \pm 219,6$ & $1391 \pm 111$ \\
\hline $\mathrm{UN}$ in plasma $\mathrm{mmol} / \mathrm{l}$ & $6,79 \pm 0,29$ & $6,8 \pm 0,49$ & $5,97 \pm 0,57$ & $5,29 \pm 0,48$ \\
\hline Urea in urine $\mathrm{mmol} / \mathrm{l}$ & $1031 \pm 48,1$ & $825 \pm 81,1^{*}$ & $840 \pm 179,7$ & $1269,2 \pm 68,5^{*}$ \\
\hline Urea excretion $\mathrm{mmol} /$ day & $13,63 \pm 1,24$ & $17,0 \pm 2,05$ & $12,5 \pm 1,15$ & $12,1 \pm 1,15$ \\
\hline Urea in plasma $\mathrm{mmol} / \mathrm{l}$ & $7,04 \pm 0,33$ & $7,18 \pm 1,13$ & $6,24 \pm 0,59$ & $6,52 \pm 0,92$ \\
\hline
\end{tabular}

3.4. L-Norvaline Induced Diuresis in the Hypertonic ISIAH Rats. In order to assess the effects of L-norvaline on fluid homeostasis in rats and correlate the treatment-related changes in BP with alterations in daily urine excretion and daily water consumption, we analyzed the mean water intake and urine output in the experimental groups. We observed significant strain and treatment-related differences in the daily urine output and water intake (Figures 4(a) and 4(b)). Of note, we did not detect any substantial differences between the groups in the basal levels of urine excretion and water intake. However, the treatment with L-norvaline was followed by a significant decline (by $17 \%$ ) in the mean daily water consumption in the normotonic rats and increase (by $29 \%$ ) in the mean urine excretion in the ISIAH rats. Nevertheless, the index, which reflects the general fluid balance and calculated by expressing urine output as a percentage of water intake, did not detect any significant differences between the groups, but a clear tendency to increase in hypertonic animals treated with L-norvaline (Figure 4(c)).
3.5. L-Norvaline Amplifies the Levels of NO Derivatives in the Plasma and Urine of Wistar but Not ISIAH Rats. To estimate the rates of NOS activity in the experimental animals, we quantified the urine and plasma concentrations of nitrites and nitrates, which are the stable metabolites of L-argininederived NO on the very last experimental day. Analysis of plasma NO metabolites (Figure 5(a)) revealed a significant $(p<0.0001)$ main effect of the treatment on the blood nitrate-nitrite concentrations. Remarkably, nitrate-nitrite contents were about 34\% higher in control normotensive animals than in hypertensive $(1.553 \pm 0.027$ vs $1.16 \pm 0.11)$. Moreover, the nitrite-nitrate levels increased significantly $(p<0.01)$ by about $35 \%$ in the normotensive group following the L-norvaline treatment. On the other hand, this parameter demonstrated a moderate (about 2.5\%) and statistically insignificant increase in ISIAH group. Two-way ANOVA was applied to check if there is an interaction between the strain and the treatment variables on the nitritenitrate concentration. The test revealed a significant effect of 


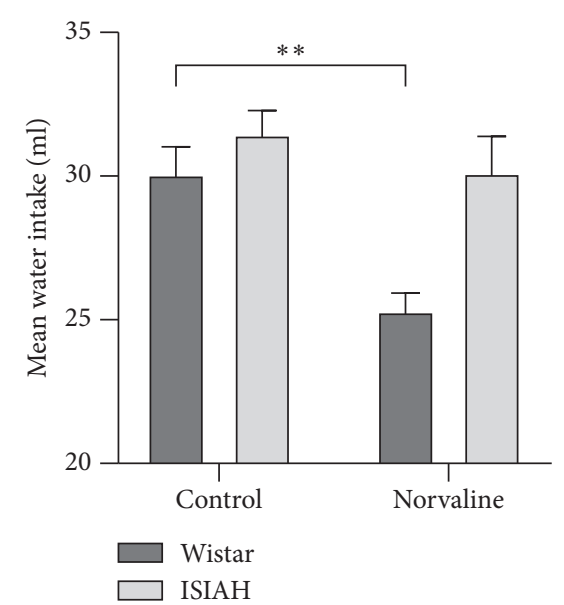

(a)

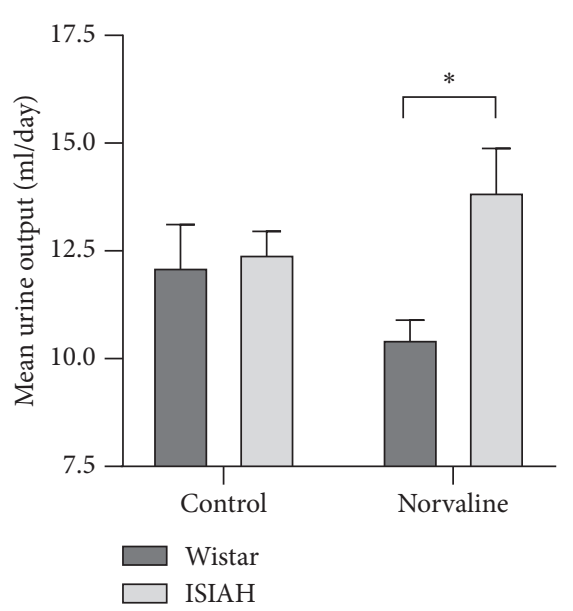

(b)

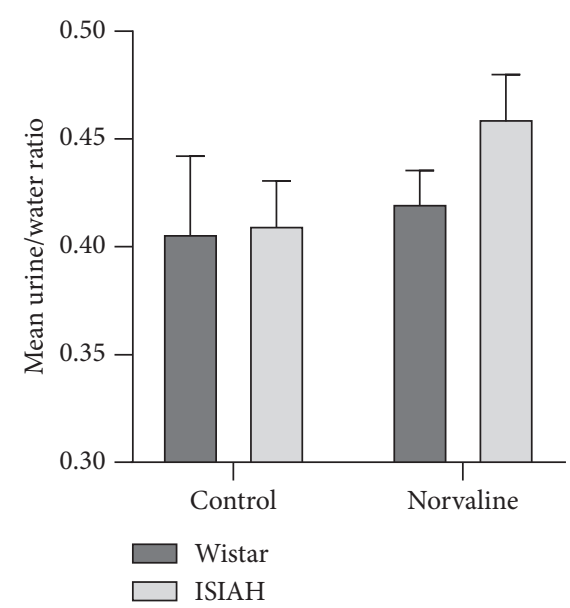

(c)

Figure 4: Effects of L-norvaline on fluid homeostasis. Mean water intake (a), mean urine output (b), and mean urine/water ratio (c). Water restriction was initiated immediately after dosing. Data presented as mean \pm SEM. Two-way ANOVA test. Significant difference is indicated as ${ }^{* *} p<0.01$ and ${ }^{*} p<0.05$.

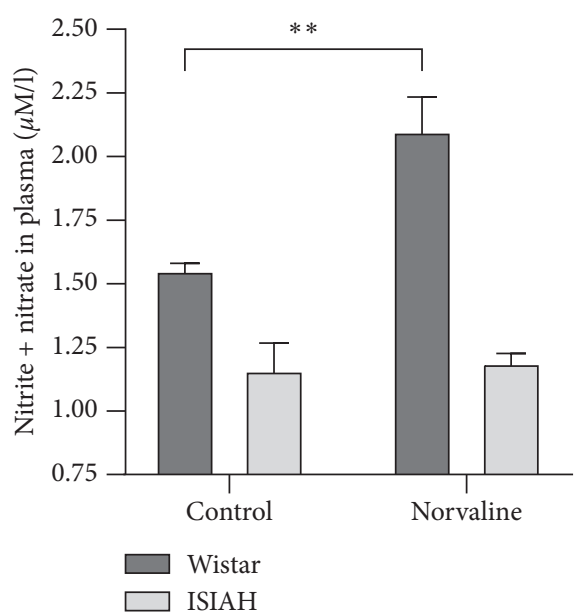

(a)

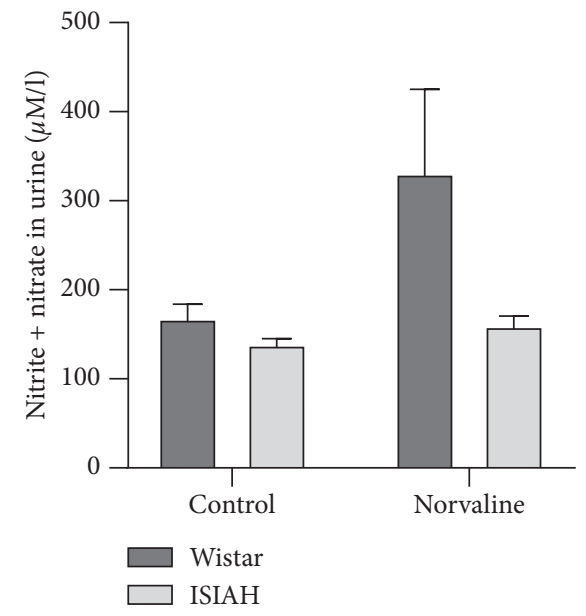

(b)

FIgURE 5: Effects of L-norvaline treatment on the levels of NO derivatives in the plasma and urine (7th experimental day). The mean concentrations of (a) plasma NO metabolites and (b) urine NO metabolites. Two-way ANOVA test; ${ }^{* *} p<0.01$; data presented as mean \pm SEM.

the strain type and the treatment upon the dependent variable. Moreover, an interaction between strain and treatment was very significant $F_{(1,17)}=9.282 p=0.0073$.

Of note, the basal content of NO metabolites in the urine is more than two orders of magnitude higher than in the blood (Figure 5(b)), and this parameter displays a substantially higher variability compared to the plasma index. Nevertheless, the plot of the urine NO derivative levels on the last experimental day demonstrate a similar pattern to the blood pattern, and the main effect of the treatment was significant ( $p=0.037)$. However, an interaction between the strain type and the treatment protocol was not statistically significant $p=0.142$. One-way ANOVA followed by Tukey's multiple comparison test revealed a significant difference just between Wistar-treated and ISIAH control.

\section{Discussion}

There is a consensus in the literature on the crucial role of arginase in the pathological processes leading to various metabolic and cardiovascular diseases, which have a mutual pathogenesis. Upregulation of ARG1, particularly, is prominent in myocardial infarction [49], diabetes [50], hypertension [51], and Alzheimer's disease [35]. ARG2 activation is a leading factor of atherosclerotic vascular disease development [52] and diabetic renal injury [53]. Accordingly, arginase inhibition has been suggested to be a promising therapeutic strategy $[20,24,54]$. Numerous preclinical investigations testing this approach have demonstrated promising results [26]. Consequently, several clinical studies have proved that inhibiting arginase with 
$\mathrm{N} \omega$-hydroxy-nor-L-arginine significantly improves microcirculation and endothelial function in diabetic patients $[55,56]$.

In the present study, we demonstrated that arginase inhibition with L-norvaline reduced BP in a rodent model of inherited stress-induced arterial hypertension but had no effect upon the BP in the normotonic animals, which accords with the previously published data showing the efficacy of another arginase inhibitor $\mathrm{N}$-hydroxy-nor-L-arginine in a murine model of hypertension [57]. Furthermore, we evidenced significantly decreased basal levels of NO derivatives in the blood of the hypertensive rats compared to the normotensive rats, which points to a decline in NOS activity in ISIAH rats and indicates seriously misbalanced relationships between arginase and NOS pathways, presumably due to L-arginine deprivation in this strain.

Surprisingly, we observed a shift in the L-arginine metabolism from arginase towards the NOS pathway with increased generation of $\mathrm{NO}$ following the L-norvaline treatment only in the normotonic group, which contrasts with the results of Bagnost et al. [57]. We suggest that in our rodent model of inherited stress-induced arterial hypertension, arginase is upregulated in a much more extreme manner than in spontaneously hypertensive rats and NOS activity is more severely suppressed. Moreover, we utilized a substantially shorter treatment protocol.

Growing evidence indicates the principal role of inflammation in the pathogenesis of hypertension, which is now considered as a low-grade inflammatory condition [58]. Hypertension is empirically characterized by the presence of numerous proinflammatory cytokines in plasma and various organs. Tumor necrosis factor- $\alpha$ (TNF$\alpha$ ), above all, has been related to hypertension development and associated with the rate of renal injury. It was established that hypertensive patients exhibit higher levels of TNF- $\alpha$ compared to normotensive individuals [59]. Likewise, the levels of TNF- $\alpha$ are significantly elevated in various rodent models of hypertension. This cytokine contributes to hypertension development in Dahl saltsensitive rats [60] and mediates hypertension in mice with angiotensin II-dependent hypertension [61]. Remarkably, the NO-deficient mouse model of hypertension is characterized by induced TNF- $\alpha$ generation and significant natriuretic response [62]. Shahid et al. elegantly demonstrated that the mice treated with a potent NOS inhibitor nitro-l-arginine exhibit substantially elevated plasma levels of TNF- $\alpha$ followed by an increase in mean arterial pressure, a decline in GFR, and a marked escalation of sodium excretion. However, pretreatment with a TNF- $\alpha$ blocker blunts the effect on the sodium excretion rate [62].

In this context, the antihypertensive effect of L-norvaline might be partially attributed to its competence in inhibiting S6K1 [34]. There are strong data showing potent anti-inflammatory properties of this substance. L-norvaline treatment leads to a drop in the microglia density in the hippocampi of Alzheimer's disease model mice, which is followed by a shift from activated to resting microglial phenotype [35]. Additionally, L-norvaline significantly reduces the brain levels of TNF- $\alpha$ in the same murine model
[40]. Therefore, its application in the models of hypertension might have a particular benefit.

In order to analyze the treatment-related changes in kidney function, we measured a list of blood and urine parameters. Even though the examination of plasma UN and creatinine levels has no adequate sensitivity for the diagnosis of renal dysfunction, the informativity of these markers could be improved by considering the effect of body weight [63]. The GFR is one of the best indices to estimate the kidney function in health and disease [43]. We calculated this parameter per unit of the body weight and did not detect any influence of the L-norvaline treatment upon the GFR in two experimental strains, which suggests a minor role of the renal filtration in the L-norvaline-associated reduction of BP.

Although L-norvaline treatment induced diuresis in the hypertensive rats, the same protocol had no impact upon the urine output in the Wistar rats. Previously, we have demonstrated that ISIAH rats show augmented levels of tubular epithelial $\mathrm{Na}$ channel-alpha compared to the normotonic Wistar rats, which can partially explain the observed phenotype [64]. Nevertheless, we speculate that this phenomenon is more related to the treatment-associated changes in the renal NO synthesis, which are dependent upon the basal levels of the renal NOS. We have disclosed earlier a characteristic significant reduction in the juxtaglomerular NOS protein levels in the ISIAH rats [64]. This deficiency leads to renal hemodynamics impairments in this model and, presumably, plays a role in the hypertensive status formation. Accordingly, L-norvaline blocks arginase and improves substrate availability for the renal NOS, and supports NO synthesis, which normalizes the kidneys' function, induces diuresis, and, subsequently, contributes to decreasing the elevated BP. Of note, inhaled NO has been shown to have a significant effect upon the renal function. Wraight and Young provided healthy middle-aged males with NO/air mixture for two hours and reported consequent increase (by about $85 \%$ ) in urine output [65].

We have mentioned above that the observed increase in the ISIAH rats' diuresis was not associated with the changes in GFR. Therefore, we speculate that the diuretic effect is related to the decrease in the sodium and water tubular reabsorption. This explanation seems plausible because NO has been shown to play a dualistic role in the regulation of renin and vasopressin secretion. Generally, NOS inhibition suppresses renin release, which indicates a stimulatory role of the L-arginine/NO pathway in the control of renin secretion [66]. However, under some conditions, and, in particular, in the models of chronic NOS inhibition, renin secretion is severely escalated $[67,68]$. This phenomenon is dependent upon factors that are not clearly understood yet, and research, identifying the precise NO regulatory role in the control of renin function and urine excretion rate, still remains to be done.

\section{Conclusions}

Our results indicate that the levels of NOS and arginase activity can be an informative biomarker to monitor the 
development and progression of clinical hypertension. Moreover, arginase inhibition, with L-norvaline in particular, possesses a serious therapeutic benefit due to a potential reduction of oxidative stress and inflammation, averting vascular dysfunction, and maintaining balanced physiological levels of NO.

Our findings prove significant BP-reducing and diuresisinducing effects of L-norvaline in a murine model of stresssensitive arterial hypertension, which suggests a potency of this agent to manage hypertension. Remarkably, a oneweek-long treatment protocol has no substantial influence upon the $\mathrm{BP}$ in the normotensive animals but leads to a meaningful BP decline in the hypertensive rats. We speculate that the observed reactions are mainly related to the immediate effects of arginase inhibition with L-norvaline upon the rate of L-arginine bioavailability, and $\mathrm{NO}$ synthesis in the kidney tissue, and the vessel wall in general. Moreover, we suggest that the effects of the treatment are strongly dependent on the initial parameters of the whole biological system, which are responsible for the regulation of renal circulation rate, and BP maintenance that are expressively misbalanced in the adult ISIAH rats [42].

Additionally, we suggest that it would be of value to investigate the contribution of the different arginase isoforms to the development of stress-induced arterial hypertension. The transgenic animals with upregulated and/or deleted arginase genes might represent attractive research models.

\section{Data Availability}

The data used to support the findings of this study are available from the corresponding author upon request.

\section{Conflicts of Interest}

The authors declare that there are no conflicts of interest regarding the publication of this paper.

\section{Authors' Contributions}

Michael Gilinsky, Arkady Markel, and Abraham O. Samson designed the experiments. Michael Gilinsky and Yulia Polityko performed the experiments. Tatyana Latysheva, Yulia Polityko, Baruh Polis, and Sergey Naumenko analyzed the data. Michael Gilinsky and Baruh Polis wrote the manuscript. Arkady Markel, Sergey Naumenko, Baruh Polis, and Abraham O. Samson advised and developed experimental methods and edited the manuscript.

\section{Acknowledgments}

Russian Foundation for Basic Research supported this work with grant no. 17-04-00916.

\section{References}

[1] B. Zhou, J. Bentham, M. Di Cesare et al., "Worldwide trends in blood pressure from 1975 to 2015: a pooled analysis of 1479 population-based measurement studies with 19.1 million participants," The Lancet, vol. 389, no. 10064, pp. 37-55, 2017.

[2] R. Merai, C. Siegel, M. Rakotz et al., "CDC grand rounds: a public health approach to detect and control hypertension," MMWR. Morbidity and Mortality Weekly Report, vol. 65, no. 45, pp. 1261-1264, 2016.

[3] H. Arima, F. Barzi, and J. Chalmers, "Mortality patterns in hypertension," Journal of Hypertension, vol. 29, pp. S3-S7, 2011.

[4] T. M. Spruill, "Chronic psychosocial stress and hypertension," Current Hypertension Reports, vol. 12, no. 1, pp. 10-16, 2010.

[5] A. Steptoe, "Psychophysiological stress reactivity and hypertension," Hypertension, vol. 52, no. 2, pp. 220-221, 2008.

[6] N. Gokce, "L-arginine and hypertension," The Journal of Nutrition, vol. 134, no. 10, pp. 2807S-2811S, 2004.

[7] A. Patel, S. Layne, D. Watts, and K. A. Kirchner, "L-arginine administration normalizes pressure natriuresis in hypertensive Dahl rats," Hypertension, vol. 22, no. 6, pp. 863-869, 1993.

[8] P. Y. Chen and P. W. Sanders, "L-arginine abrogates saltsensitive hypertension in Dahl/Rapp rats," Journal of Clinical Investigation, vol. 88, no. 5, pp. 1559-1567, 1991.

[9] T. C. Wascher, K. Posch, S. Wallner, A. Hermetter, G. M. Kostner, and W. F. Graier, "Vascular effects of L-arginine: anything beyond a substrate for the NO-synthase?" Biochemical and Biophysical Research Communications, vol. 234, no. 1, pp. 35-38, 1997.

[10] S. Vasdev and V. Gill, "The antihypertensive effect of arginine," International Journal of Angiology, vol. 17, no. 1, pp. 07-22, 2008.

[11] B. Tong and A. Barbul, "Cellular and physiological effects of arginine," Mini-Reviews in Medicinal Chemistry, vol. 4, no. 8, pp. 823-832, 2004.

[12] C. Zhang, T. W. Hein, W. Wang et al., "Upregulation of vascular arginase in hypertension decreases nitric oxidemediated dilation of coronary arterioles," Hypertension, vol. 44, no. 6, pp. 935-943, 2004.

[13] R. M. J. Palmer, “The l-arginine," Current Opinion in $\mathrm{Ne}$ phrology and Hypertension, vol. 2, no. 1, pp. 122-128, 1993.

[14] U. Förstermann, N. Xia, and H. Li, "Roles of vascular oxidative stress and nitric oxide in the pathogenesis of atherosclerosis," Circulation Research, vol. 120, no. 4, pp. 713-735, 2017.

[15] H. Li and U. Förstermann, "Uncoupling of endothelial NO synthase in atherosclerosis and vascular disease," Current Opinion in Pharmacology, vol. 13, no. 2, pp. 161-167, 2013.

[16] J. W. Knowles, R. L. Reddick, J. C. Jennette, E. G. Shesely, O. Smithies, and N. Maeda, "Enhanced atherosclerosis and kidney dysfunction in eNOS-/-Apoe-/- mice are ameliorated by enalapril treatment," Journal of Clinical Investigation, vol. 105, no. 4, pp. 451-458, 2000.

[17] A. J. Lusis, "Atherosclerosis," Nature, vol. 407, no. 6801, pp. 233-241, 2000.

[18] W. Durante, F. K. Johnson, and R. A. Johnson, “Arginase: a critical regulator of nitric oxide synthesis and vascular function," Clinical and Experimental Pharmacology and Physiology, vol. 34, no. 9, pp. 906-911, 2007.

[19] G. Wu and S. M. Morris, "Arginine metabolism: nitric oxide and beyond," Biochemical Journal, vol. 336, no. 1, pp. 1-17, 1998.

[20] B. Polis and A. O. Samson, "Arginase as a potential target in the treatment of alzheimer's disease," Advances in Alzheimer's Disease, vol. 7, no. 4, pp. 119-140, 2018.

[21] S. Ryoo, A. Bhunia, F. Chang, A. Shoukas, D. E. Berkowitz, and L. H. Romer, "OxLDL-dependent activation of arginase II 
is dependent on the LOX-1 receptor and downstream RhoA signaling," Atherosclerosis, vol. 214, no. 2, pp. 279-287, 2011.

[22] D. Pandey, A. Bhunia, Y. J. Oh et al., "OxLDL triggers retrograde translocation of arginase 2 in aortic endothelial cells via ROCK and mitochondrial processing peptidase," Circulation Research, vol. 115, no. 4, pp. 450-459, 2014.

[23] J. Frostegård, R. Wu, C. Lemne, T. Thulin, J. L. Witztum, and U. de Faire, "Circulating oxidized low-density lipoprotein is increased in hypertension," Clinical Science, vol. 105, no. 5, pp. 615-620, 2003.

[24] J. Pernow and C. Jung, "Arginase as a potential target in the treatment of cardiovascular disease: reversal of arginine steal?" Cardiovascular Research, vol. 98, no. 3, pp. 334-343, 2013.

[25] H. M. El-Bassossy, R. El-Fawal, A. Fahmy, and M. L. Watson, "Arginase inhibition alleviates hypertension in the metabolic syndrome," British Journal of Pharmacology, vol. 169, no. 3, pp. 693-703, 2013.

[26] K. J. Peyton, X.-M. Liu, A. R. Shebib, F. K. Johnson, R. A. Johnson, and W. Durante, "Arginase inhibition prevents the development of hypertension and improves insulin resistance in obese rats," Amino Acids, vol. 50, no. 6, pp. 747754, 2018.

[27] T. Bagnost, L. Ma, R. F. Da Silva et al., "Cardiovascular effects of arginase inhibition in spontaneously hypertensive rats with fully developed hypertension," Cardiovascular Research, vol. 87, no. 3, pp. 569-577, 2010.

[28] M. V. Pokrovskiy, M. V. Korokin, S. A. Tsepeleva et al., "Arginase inhibitor in the pharmacological correction of endothelial dysfunction," International Journal of Hypertension, vol. 2011, Article ID 515047, 4 pages, 2011.

[29] R. Rognstad, "Sources of ammonia for urea synthesis in isolated rat liver cells," Biochimica et Biophysica Acta (BBA) General Subjects, vol. 496, no. 2, pp. 249-254, 1977.

[30] T. Saheki, Y. Sato, S. Takada, and T. Katsunuma, "Regulation of urea synthesis in rat liver," The Journal of Biochemistry, vol. 86, no. 3, pp. 745-750, 1979.

[31] C.-I. Chang, J. C. Liao, and L. Kuo, "Arginase modulates nitric oxide production in activated macrophages," American Journal of Physiology-Heart and Circulatory Physiology, vol. 274, no. 1, pp. H342-H348, 1998.

[32] A. M. Bode, J. D. Foster, and R. C. Nordlieon, "Glycogenesis from glucose and ureagenesis in isolated perfused rat livers," Biochemistry, vol. 269, no. 11, pp. 7879-7886, 1994.

[33] J. Dumont, D. Meroufel, C. Bauters et al., "Association of ornithine transcarbamylase gene polymorphisms with hypertension and coronary artery vasomotion," American Journal of Hypertension, vol. 22, no. 9, pp. 993-1000, 2009.

[34] X.-F. Ming, A. G. Rajapakse, J. M. Carvas, J. Ruffieux, and Z. Yang, "Inhibition of S6K1 accounts partially for the antiinflammatory effects of the arginase inhibitor L-norvaline," BMC Cardiovascular Disorders, vol. 9, no. 1, Article ID 12, 2009.

[35] B. Polis, K. D. Srikanth, E. Elliott, H. Gil-Henn, and A. O. Samson, "L-norvaline reverses cognitive decline and synaptic loss in a murine model of alzheimer's disease," Neurotherapeutics, vol. 15, no. 4, pp. 1036-1054, 2018.

[36] Q. N. Dinh, G. R. Drummond, C. G. Sobey, and S. Chrissobolis, "Roles of inflammation, oxidative stress, and vascular dysfunction in hypertension," BioMed Research International, vol. 2014, Article ID 406960, 11 pages, 2014.

[37] H. Tomiyama, K. Shiina, C. Matsumoto-Nakano et al., "The contribution of inflammation to the development of hypertension mediated by increased arterial stiffness," Journal of the American Heart Association, vol. 6, no. 7, 2017.

[38] V. Kumar, C. Wollner, T. Kurth, J. D. Bukowy, and A. W. Cowley, "Inhibition of mammalian target of rapamycin complex 1 attenuates salt-induced hypertension and kidney injury in dahl salt-sensitive rats," Hypertension, vol. 70, no. 4, pp. 813-821, 2017.

[39] A. De, M. F. Singh, V. Singh, V. Ram, and S. Bisht, “Treatment effect of 1-Norvaline on the sexual performance of male rats with streptozotocin induced diabetes," European Journal of Pharmacology, vol. 771, pp. 247-254, 2016.

[40] B. Polis, K. Srikanth, V. Gurevich, H. Gil-Henn, and A. Samson, "L-Norvaline, a new therapeutic agent against alzheimer's disease," Neural Regeneration Research, vol. 14, no. 9, p. 1562, 2019.

[41] O. E. Redina and A. L. Markel, "Stress, genes, and hypertension. Contribution of the ISIAH rat strain study," Current Hypertension Reports, vol. 20, no. 8, p. 66, 2018.

[42] A. A. Seryapina, O. B. Shevelev, M. P. Moshkin, A. L. Markel, and A. E. Akulov, "Stress-sensitive arterial hypertension, haemodynamic changes and brain metabolites in hypertensive ISIAH rats: MRI investigation," Experimental Physiology, vol. 102, no. 5, pp. 523-532, 2017.

[43] A. S. Levey, L. A. Stevens, C. H. Schmid et al., "A new equation to estimate glomerular filtration rate," Annals of Internal Medicine, vol. 150, no. 9, pp. 604-612, 2009.

[44] Y.-c. Lin, N. Bansal, E. Vittinghoff, A. S. Go, and C.-y. Hsu, "Determinants of the creatinine clearance to glomerular filtration rate ratio in patients with chronic kidney disease: a cross-sectional study," BMC Nephrology, vol. 14, no. 1, p. 268, 2013.

[45] T. Rassaf, M. Preik, P. Kleinbongard et al., "Evidence for in vivo transport of bioactive nitric oxide in human plasma," Journal of Clinical Investigation, vol. 109, no. 9, pp. 1241-1248, 2002.

[46] S. Shiva, X. Wang, L. A. Ringwood et al., "Ceruloplasmin is a NO oxidase and nitrite synthase that determines endocrine NO homeostasis," Nature Chemical Biology, vol. 2, no. 9, pp. 486-493, 2006.

[47] L. Liaudet, F. G. Soriano, and C. Szabó, "Biology of nitric oxide signaling," Critical Care Medicine, vol. 28, no. 4, pp. N37-N52, 2000.

[48] A. L. Markel, O. E. Redina, M. A. Gilinsky et al., "Neuroendocrine profiling in inherited stress-induced arterial hypertension rat strain with stress-sensitive arterial hypertension," Journal of Endocrinology, vol. 195, no. 3, pp. 439-450, 2007.

[49] C. Jung, A. T. Gonon, P.-O. Sjoquist, J. O. Lundberg, and J. Pernow, "Arginase inhibition mediates cardioprotection during ischaemia-reperfusion," Cardiovascular Research, vol. 85, no. 1, pp. 147-154, 2010.

[50] M. J. Romero, D. H. Platt, H. E. Tawfik et al., "Diabetes-induced coronary vascular dysfunction involves increased arginase activity," Circulation Research, vol. 102, no. 1, pp. 95-102, 2008.

[51] H. A. Toque, K. P. Nunes, M. Rojas et al., “Arginase 1 mediates increased blood pressure and contributes to vascular endothelial dysfunction in deoxycorticosterone acetate-salt hypertension," Frontiers in Immunology, vol. 4, p. 219, 2013.

[52] S. Ryoo, D. E. Berkowitz, and H. K. Lim, "Endothelial arginase II and atherosclerosis," Korean Journal of Anesthesiology, vol. 61, no. 1, pp. 3-11, 2011. 
[53] S. M. Morris, T. Gao, T. K. Cooper, D. Kepka-Lenhart, and A. S. Awad, "Arginase-2 mediates diabetic renal injury," Diabetes, vol. 60, no. 11, pp. 3015-3022, 2011.

[54] S. Ryoo, G. Gupta, A. Benjo et al., "Endothelial arginase II," Circulation Research, vol. 102, no. 8, pp. 923-932, 2008.

[55] A. Shemyakin, O. Kövamees, A. Rafnsson et al., "Arginase inhibition improves endothelial function in patients with coronary artery disease and type 2 diabetes mellitus," Circulation, vol. 126, no. 25, pp. 2943-2950, 2012.

[56] O. Kövamees, A. Shemyakin, A. Checa et al., "Arginase inhibition improves microvascular endothelial function in patients with type 2 diabetes mellitus," The Journal of Clinical Endocrinology \& Metabolism, vol. 101, no. 11, pp. 3952-3958, 2016.

[57] T. Bagnost, A. Berthelot, M. Bouhaddi et al., "Treatment with the arginase inhibitor $\mathrm{N} \omega$-hydroxy-nor-L-arginine improves vascular function and lowers blood pressure in adult spontaneously hypertensive rat," Journal of Hypertension, vol. 26, no. 6, pp. 1110-1118, 2008.

[58] E. Mehaffey and D. S. A. Majid, "Tumor necrosis factor- $\alpha$, kidney function, and hypertension," American Journal of Physiology-Renal Physiology, vol. 313, no. 4, pp. F1005-F1008, 2017.

[59] N. R. Barbaro, T. M. de Araújo, J. E. Tanus-Santos, G. F. Anhê, V. Fontana, and H. Moreno, "Vascular damage in resistant hypertension: TNF-alpha inhibition effects on endothelial cells," BioMed Research International, vol. 2015, Article ID 631594, 8 pages, 2015.

[60] B. Huang, Y. Cheng, K. Usa et al., "Renal tumor necrosis factor $\alpha$ contributes to hypertension in dahl salt-sensitive rats," Scientific Reports, vol. 6, no. 1, Article ID 21960, 2016.

[61] J. Zhang, M. B. Patel, R. Griffiths et al., "Tumor necrosis factor- $\alpha$ produced in the kidney contributes to angiotensin IIdependent hypertension," Hypertension, vol. 64, no. 6, pp. 1275-1281, 2014.

[62] M. Shahid, J. Francis, K. Matrougui, and D. S. A. Majid, "Involvement of tumor necrosis factor- $\alpha$ in natriuretic response to systemic infusion of nitric oxide synthase inhibitor in anesthetized mice," American Journal of Physiology-Renal Physiology, vol. 299, no. 1, pp. F217-F224, 2010.

[63] Y. Miyagawa, N. Takemura, and H. Hirose, "Assessments of factors that affect glomerular filtration rate and indirect markers of renal function in dogs and cats," Journal of Veterinary Medical Science, vol. 72, no. 9, pp. 1129-1136, 2010.

[64] S. Amstislavsky, P. Welker, J.-H. Frühauf et al., "Renal and endocrine changes in rats with inherited stress-induced arterial hypertension (ISIAH)," Histochemistry and Cell Biology, vol. 125, no. 6, pp. 651-659, 2006.

[65] W. M. Wraight and J. D. Young, "Renal effects of inhaled nitric oxide in humans," British Journal of Anaesthesia, vol. 86, no. 2, pp. 267-269, 2001.

[66] I. A. Reid, "Role of nitric oxide in the regulation of renin and vasopressin secretion," Frontiers in Neuroendocrinology, vol. 15, no. 4, pp. 351-383, 1994.

[67] M. O. Ribeiro, E. Antunes, G. De Nucci, S. M. Lovisolo, and R. Zatz, "Chronic inhibition of nitric oxide synthesis. A new model of arterial hypertension," Hypertension, vol. 20, no. 3, pp. 298-303, 1992.

[68] F. J. Salazar, J. M. Pinilla, F. López, J. C. Romero, and T. Quesada, "Renal effects of prolonged synthesis inhibition of endothelium-derived nitric oxide," Hypertension, vol. 20, no. 1, pp. 113-117, 1992. 